\title{
A Review on Feature Extraction Techniques and General Approach for Face Recognition
}

\author{
Aakanksha Agrawal \\ Department of CSE \\ RCET, Bhilai \\ CG, India
}

\author{
Steve Samson \\ Department of CSE \\ RCET, Bhilai \\ CG, India
}

\begin{abstract}
In recent time, alongwith the advances and new inventions in science and technology, fraud people and identity thieves are also becoming smarter by finding new ways to fool the authorization and authentication process. So, there is a strong need of efficient face recognition process or computer systems capable of recognizing faces of authenticated persons. One way to make face recognition efficient is by extracting features of faces. Several feature extraction techniques are available such as template based, appearancebased, geometry based, color segmentation based, etc. This paper presents an overview of various feature extraction techniques followed in different reasearches for face recognition in the field of digital image processing and gives an approach for using these feature extraction techniques for efficient face recognition.
\end{abstract}

Keywords: face recognition, feature extraction, lip extraction, eye extraction

\section{INTRODUCTION}

Feature Extraction is a kind of process for reducing dimensionality so as to represent the interesting image parts with great efficiency as a compact feature vector [16]. This is useful in case of large images.

No one can say which algorithm is best suitable for extracting features. The algorithm selection is dependent on: (1) What exactly is the task it needs to perform (2) Whether supervised method is needed or unsupervised (3) Whether Inexpensive method is required or strong computational method is required etc. Some techniques for feature extraction are Speeded Up Robust Features (SURF), Histogram of Oriented Gradients (HOG), Color Histograms, Local Binary Patterns (LBP), Haar wavelets, etc. [16]

Many software packages for data analysis are available providing feature extraction and dimension reduction. Some numerical programming environments such as MATLAB, NumPy, SciLab also provides technique for feature extraction like Principal Component analysis (PCA) etc. [18]

\section{FACE FEATURES FOR EXTRACTION}

\subsection{Lip Feature}

Lip is a sensory organ existing in visible portion of human face and considered to be different for each and every individual. There are researches carried out for face recognition and classification of gender using lip shape and color analysis. [3]

\subsection{Eye/Iris Feature}

Eye/Iris has randomness due to its small tissues which provides differentiation to the pattern of eye for each and every individual human being. [13] The stableness, uniqueness and non-invasion, these qualities make the iris outstanding among several biometric features. [14]

\subsection{Nose Feature}

The nose tip is a distinctive point of human face. It also remains unaffected even due to changes in facial expressions. [18] Thus, it proves to be efficient for face recognition.

\section{TECHNIQUES FOR FEATURE EXTRACTION PURPOSE}

\subsection{Face Part Detection (FPD)}

Convolution technique is used in this algorithm. It works by multiplying vectors and returns values by using length and width. Gabor features are done using Gabor filters and here image decomposition is done by converting real part and imaginary part. [12]

\subsection{Principal Component Analysis (PCA)}

In this, the dimensionality is reduced by projecting the data onto the largest eigenvectors. [18] It selects the weights on the basis of frequency in the frequency domain. It cannot separate the class linearly. 


\subsection{Linear Discriminant Analysis (LDA)}

LDA is a generalization of Fisher's linear discriminant to find a linear combination of features that characterizes two or more classes of events. The resulting combination may be used for dimensionality Reduction or as a linear classifier. [18]

\subsection{Speeded Up Robust Features (SURF)}

Surf algorithm is an improvement of Scale Invariant Feature Transform (SIFT) algorithm. Surf uses a fast multi-scale Hessian keypoint detector that can find keypoints. It can also be used to compute user specified keypoints. Only 8 bit grayscale images are supported. [3]

\section{RELATED WORK}

4.1 Rutuja G. Shelke, S.A.Annadate presented a novel approach for Face Recognition and Gender classification strategy using the features of lips. Here feature extraction is carried out by using Principal component analysis (PCA) and Gabor wavelet. Out of two techniques, results of Gabor filter are more accurate and fast because it is having less leakage in time frequency domain. [International Journal of Innovation and Scientific Research (IJISR), Vol 10, No.2, Oct.2014, Innovative Space of Scientific Research Journals (ISSR)].

4.2 Ishvari S. Patel, Apurva A. Desai used Preprocessing techniques like Edge Detection by Canny Method and Height and Width comparison for Lip Contour Detection. This model works effectively and gives around 98\% result for image sequences but we can still improve accuracy of result by extracting perfect lips region. [International Journal of Scientific Research (IJSR), Volume II, Issue V, May 2013].

4.3 Sambit Bakshi, Rahul Raman, Pankaj K Sa paper proposes that grayscale lip images constitute local features. The claim has been experimentally established by extracting local features applying two techniques viz. SIFT and SURF. The results obtained are enough to establish that unique local features exist in lip images through which an individual can be recognized. [India Conference (INDICON), 2011 Annual IEEE].

4.4 Sasikumar Gurumurty, B. K. Tripathy divided methodology into: Mouth Region Localization and Key point's Extraction and Model Fitting. In first and second steps, mouth region and key points are found by using hybrid edges, which combine color and intensity information. In third step, cubic polynomial models are fitted using key points position and hybrid edges. An automatic, robust and accurate lip segmentation method has been presented. This is considered as good result and encourage for its use combined with other biometrics systems.[ I.J. Intelligent Systems and Applications (IJISA), July 2012 in MECS].

4.5 B. Sangeetha Devi, V.J.Arul Karthick used two processes for lip recognition. First, face detection by Viola and Jones algorithm. Second, lip detection by morphological operation and five various mouth corner points. Lip biometric can be used to authenticate an individual since the lip is unique. [International Journal of Advanced Research Trends in Engineering and Technology (IJARTET), Vol. II, Special Issue I, March 2015].

\section{GENERAL APPROACH FOR FACE RECOGNITION}

\subsection{Acquiring the image of an individual's face \\ Digitally scan an existing photograph, or Acquire a live picture of a subject.}

\subsection{Locate image of face}

Software is used to locate the faces in the image that has been obtained.

\subsection{Analysis of facial image}

Software measures face according to its peaks and valleys and focuses on the inner area of the face.

\subsection{Comparison}

The face print created by the software is compared to all face prints the system has stored in its database.

\subsection{Match or No Match}

Software decides whether or not any comparisons from the above step are close enough to declare a possible match.

\section{PERFORMANCE EVALUATION PARAMETERS}

\subsection{False Acceptance Rate (FAR)}

The probability that a system will incorrectly identify an individual or will fail to reject an imposter. (Also called Type2 Error Rate)

\section{FAR=NFA/NIIA}

$N F A=$ number of false acceptance

NIIA=number of imposter identification attempts

\subsection{False Rejection Rate (FRR)}

The probability that a system will fail to identify an enrolee. (Also called Type1 Error Rate)

$N F A=$ number of false rejection

FRR $=$ NFR/NEIA

$N I I A=$ number of enrolee identification attempts

\section{SCOPE OF FUTURE WORK}

Face Recognition is a very vast and elaborated field. It has no end. As the advancement in science and technology, new techniques will continue developing day-by-day. Today, Lip and Eye extraction are mostly discussed techniques for face recognition purpose but in future many more advance techniques will arise for performing Face Recognition with much more accuracy and efficiency.

\section{ACKNOWLEDGMENTS}

Our sincere thanks to all the respected and experienced faculties for their valuable guidance and motivation that always encouraged us to give our full dedication towards a new improvement in the field of science and image processing. 


\section{REFERENCES}

[1] Rutuja G. Shelke and S. A. Annadate, "Face Recognition and Gender Classification Using Feature of Lips", International Journal of Innovation and Scientific Research (IJISR), Innovative Space of Scientific Research Journals (ISSR), Vol. 10, No. 2, Oct. 2014.

[2] B. Sangeetha Devi and V. J. Arul Karthick, "Lip Recognition With Support Vector Machine (SVM) Classifier", International Journal of Advanced Research Trends in Engineering and Technology (IJARTET), Vol. II, Special Issue I, March 2015.

[3] Sambit Bakshi, Rahul Raman, Pankaj K Sa, "Lip pattern recognition based on local feature extraction", India Conference (INDICON), IEEE Annual, 2011.

[4] Sunil Sangve, Nilakshi Mule, "Lip Recognition for Authentication and Security", IOSR Journal of Computer Engineering (IOSR-JCE) Volume 16, Issue 3, Ver. VII, MayJun. 2014.

[5] Ishvari S. Patel and Apurva A. Desai, "Lip Segmentation Based on Edge Detection Technique", International Journal of Scientific Research (IJSR), Volume II, Issue V, May 2013.

[6] Duy Nguyen, David Halupka, Parham Aarabi and Ali Sheikholeslami, "Real-Time Face Detection and Lip Feature Extraction Using Field-Programmable Gate Arrays", IEEE Transactions on Systems, Man and Cybernetics-Part B: Cybernetics, Vol. 36, No.4, August 2006.

[7] Sasikumar Gurumurty and B. K. Tripathy, "Design and Implementation of Face Recognition System in Matlab Using the Features of Lips", I.J. Intelligent Systems and Applications (IJISA), July 2012 in MECS.

[8] Jyoti Bedre and Shubhangi Sapkal, "Comparative Study of Face Recognition Techniques", Emerging Trends in Computer Science and Information Technology (ETCSIT2012), International Journal of Computer Applications (IJCA), 2012.
[9] Riddhi Patel and Shruti B. Yagnik, "A Literature Survey on Face Recognition Techniques", International Journal of Computer Trends and Technology (IJCTT), Volume 5, No.4, Nov 2013.

[10] John Daugman, "How Iris Recognition works", IEEE Transactions on Circuits and Systems for Video Technology, Volume. 14, No. 1, Jan 2004.

[11] Mayank Vatsa, Richa Singh, and Afzel Noore, "Improving Iris Recognition Performance Using Segmentation, Quality Enhancement, Match Score Fusion, and Indexing", IEEE Transactions on Systems, Man and Cybernetics- Part B: Cybernetics, Feb 2008.

[12] Dr. S. Vijayarani, S. Priyatharsini, "Facial Feature Extraction Based On FPD and GLCM Algorithms", International Journal of Innovative Research in Computer and Communication Engineering, Vol.3, Issue 3, March 2015.

[13] Pankaj K.Sa, S.S. Barpanda, Bansidhar Manjhi, "Region Based Feature Extraction from Non-Cooperative Iris Images", Innovation Syst Software Engg, 2015.

[14] Changcheng Li, Weidong Zhou, Shasha Yuan, "Iris Recognition Based on a Novel Variation of Local Binary Pattern", Springer-Verlag Berlin Hiedelberg, Vis Comput, 2014.

[15] Rafael C. Gonzalez, Richard E. Woods and Steven L. Eddins, "Digital Image Processing Using MATLAB", Second Edition

[16] The MathWorks, Inc., "Image Processing Toolbox", User's Guide, COPYRIGHT 1993-2015

[17] S. N. Sivanandam, S. Sumathi, S. N. Deepa "Neural Network using MATLAB 6.0".

[18] www.google.com 